\title{
Dynamic feature for an effective elbow-joint angle estimation based on electromyography signals
}

\author{
Triwiyanto, Triana Rahmawati, Endro Yulianto, Muhammad Ridha Mak'ruf, \\ Priyambada Cahya Nugraha \\ Department of Electromedical Engineering, POLTEKKES KEMENKES Surabaya, Indonesia
}

\begin{abstract}
Article Info
Article history:

Received Feb 29, 2019

Revised Dec 30, 2019

Accepted Jan 14, 2020

\section{Keywords:}

\section{Adaptive}

EMG

Estimation

Non-pattern

Wilson

ABSTRACT

Some physical parameters influence the electromyography signal (EMG). when the EMG signal is used to estimate the position of the elbow. An adaptable feature was important to reduce a variation on the parameters. The aim of this paper is to estimate the joint position of the elbow using EMG signal based on a dynamic function. The major contribution of this work is that the method proposed is capable of determining the elbow position using the non-pattern (NPR) recognition (PR) method. A Wilson amplitude (WAMP) which used a dynamic threshold was used to reduce the EMG signal. The dynamic threshold was generated from the root mean square (RMS) processor. With the dynamic threshold, the model could adapt to any variations on the independent variables. In order to confirm this opportunity, this work involved ten healthy male subjects to perform an experimental protocol. After a tuning and calibration process, the mean of RMS error and correlation coefficient are $9.83^{\circ} \pm 1.69^{\circ}$ and $0.98 \pm 0.01$ for a single cycle of motion, $10.39^{\circ} \pm 1.82^{\circ}$ and $0.97 \pm 0.01$ for a continuous cycle of motion and $15.19^{\circ} \pm 1.92^{\circ}$ and $0.94 \pm 0.02$ for the arbitrary gesture. For conclusion, the performance of the prediction did not significantly depend on the varying cycle of gesture (p-value >0.05). This study has confirmed that the success of the non-pattern recognition-based prediction of elbow position is adaptable to any different subjects, loads, and speed of motion.
\end{abstract}

Copyright $(2020$ Institute of Advanced Engineering and Science. All rights reserved.

\section{Corresponding Author:}

Triwiyanto,

Department of Electromedical Engineering,

POLTEKKES KEMENKES Surabaya,

Jl. Pucang Jajar Timur No.10, Surabaya, Jawa Timur, Indonesia.

Email: triwiyanto123@gmail

\section{INTRODUCTION}

Electromyography (EMG) can represent the muscle activities of the human limb. The EMG is widely used in the myoelectric control in the exoskeleton and prosthetic for rehabilitation or assistive devices [1]. As discussed in the literature review, the joint of the limb can be predicted using surface electromyography signal. In general, the EMG-based joint angle estimation algorithm is divided into two types, namely: pattern recognition (PR) and no-pattern recognition (NPR) based methods [2]. Commonly PR method used machine learning to train the system, however, in the NPR method, it used filtering technique or optimization method. Several efforts have been made to estimate a joint angle at the upper limb or lower limb based on the EMG signal in those two categories. Li suggested a PR approach for estimating the lower joint angle of the limbs (knee and hip) using the least square support vector machine (LS-SVM) [3]. Hill-based musculoskeletal models are often used to approximate the position of elbow (for flexion and extension motion) and force [4] using electromyography signal. Pau et al. resulted in a high performance on the prediction of the elbow position for a single cycle [5]. However, the mean RMSE decreased when the complexities of the movement increased (at the continuous and random movement). One tool in the pattern recognition category is artificial neural 
network (ANN), which is often used to identify the characteristics of electromyography and to overcome a nonlinearity relationship between the EMG signal and the joint angle. Tang et al. created an EMG-signal based model for estimating the position of elbow using ANN with backpropagation algorithm [6]. Nonetheless, to improve the accuracy of the calculation for different loads, they combined the EMG signal and a load cell sensor as ANN inputs to identify load variations on the forearm. Previous studies mainly proposed a method for improving estimation accuracy based on machine learning with PR methods such as ANN, SVM and Genetic Algorithm [7]. However, the limitations of the pattern recognition method were that it only recognized the variables, such as speed, type of movement and subjects that had been trained and could not identify a new-added variable. It was also still a problem how to develop a model that could estimate the elbow-joint angle for varying variables with only using the EMG signal and without using additional sensors, such as accelerometer [8] and force sensor [6] which were to increase the performance of the prediction. Besides using the PR approach, separate earlier studies used the NPR method to study the relationship between the EMG and the elbow location. The advantage of using this method is that the non-pattern recognition method does not require a training process to recognize the relationship between the EMG and angle, furthermore, it can be used generally for different variables. Lee et al. predicted a movement of a lifting task using a time-domain analysis [9]. They found that the mean correlation coefficient was about 0.82 . However, they reported that the correlation coefficient was lower for a light object and high speed. Jang et al. used a spring-damper pendulum model to measure the shoulder joint for flexion movement based on an EMG signal [10]. By extracting the EMG signal into the function, Yu et al. used the EMG signal to predict the position of the elbow. A low-pass filter was added to find a smooth estimation. However, those previous studies did not evaluate the model with varying types of movements and speeds of motion [11]. These variables were very important to be evaluated so that the model could approach the human elbow function naturally.

There is some limitation mentioned in the previous research; therefore, when a different type of motion (continuous and random movements) is performed and the problem is solved in adaptation when the model is performed at different speeds and types of movements, a new method must be presented to answer the problem in precision. This paper presents the NPR approach for predicting the elbow position directly using the EMG signal extraction function and the filtering technique. The purpose of this paper is to propose a system for predicting the angle of the elbow joint, using muscle biceps. In addition, the proposed approach can be adjusted to any situations, such as varying speeds and movement types, using a dynamic threshold-based function of EMG. This paper is constructed into five sections. Section II explains the materials, the proposed method, and step to estimate the elbow-joint angle. Section III explains the study results and the conclusion. The findings of this work are shown in Section IV. Finally, Section V explains this study's conclusion.

\section{METHODS}

\subsection{Subjects}

In this study, we invited ten young people to participate in the data acquisition (Age range: 20-21 years old, weight range: $50-70 \mathrm{~kg}$ ). The experimental procedures were explained to the participant before the experiment was executed. An inform consent form was given to the subject so that they can read and understand the aim of this study. The experimental procedures were reviewed and approved by the Polytechnic of Health Surabaya Ethics Committee, Ministry of Health Indonesia. The participants were selected who had no injury and no prior muscle diseases problem. In the data recording, the subjects were trained to follow the sequence of the experiment with various tools and windows application programs.

\subsection{Procedures}

Participants are in the upright position and wearing the exoskeleton in the data recording process Figure 1. The elbow movement was synchronized with the exoskeleton frame. A linear potentiometer was used to measure the real position of the elbow which it was used as standard value. Previously, the output of the potentiometer has been calibrated using a digital goniometer (Mini digital protactometer, SCMMC, SC810-100, China). Participants were prepared to move the elbow in the 0o to 1450 bending and extension range. The movements were guided using a customized metronome program that was developed using a visual programming language based on Windows operating system. The metronome was used to direct the subjects by a trial form in single, continuous and spontaneous motions with varying motion duration (6 seconds, 8 seconds, and 10 seconds). In this study, under three different periods, the subjects performed flexion and extension movements P1 (6s), P2 (8s), P3 (10s), and three different types of motion M1 (single cycle), M2 (continuous cycle) and M3 (random cycle). To prevent the effect of muscle fatigue, every subsequence of the trial, the subjects were given 5 minutes of rest time. 


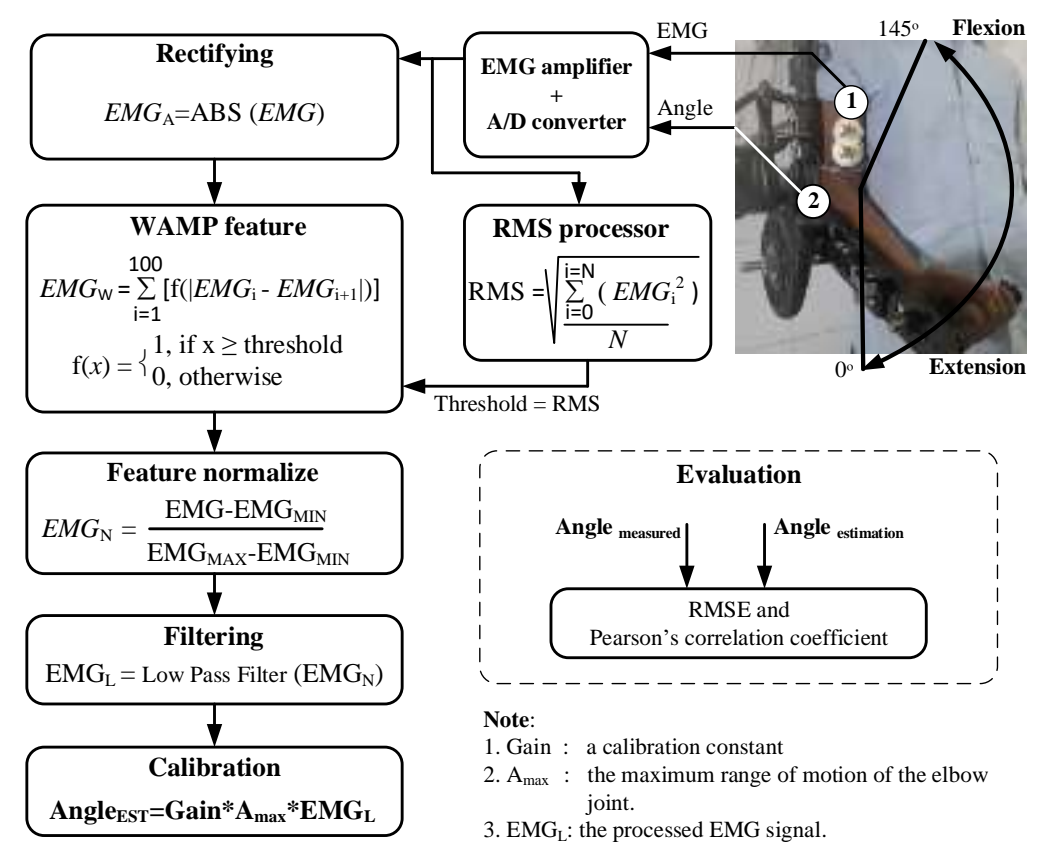

Figure 1. Modelling and calibration of elbow-joint angle estimation using WAMP parameter

\subsection{Data recording}

The EMG signal was gathered using an AD620-based bio amplifier with a built-in preamplifier. An analog Butterworth filter with a cut-off frequency between 20 and $500 \mathrm{~Hz}$ was added. This is in according to the EMG characteristics [12]. To eliminate the power line noise which joint into the EMG signal, then, notch filter 50 $\mathrm{Hz}$ was applied. In order to offset the EMG level, the summing amplifier was applied. Two pair standard disposable electrodes as used in the ECG data recording was used in the EMG data recording. The space between electrode pair, which placed on the surface of the biceps and triceps muscle, was $20 \mathrm{~mm}$. Biceps and triceps are the muscles that are most active for these movements in the process of flexion and extension [13]. The potentiometer was placed in the spot between the upper arm and forearm of the exoskeleton frame which used to measure the real position of the elbow.

\subsection{Data processing}

For every 100 samples, the recorded data (EMG and angle) were extracted using adjacent windowing techniques as recommended in the previous study recorded data (EMG and angle) $[14,15]$. In the other part of our analysis, the efficiency of the extraction of time-domain features was assessed [16]. WAMP is the number of times the difference between two neighboring EMG samples exceeding the maximum value. The WAMP is defined in the following (1) [16].

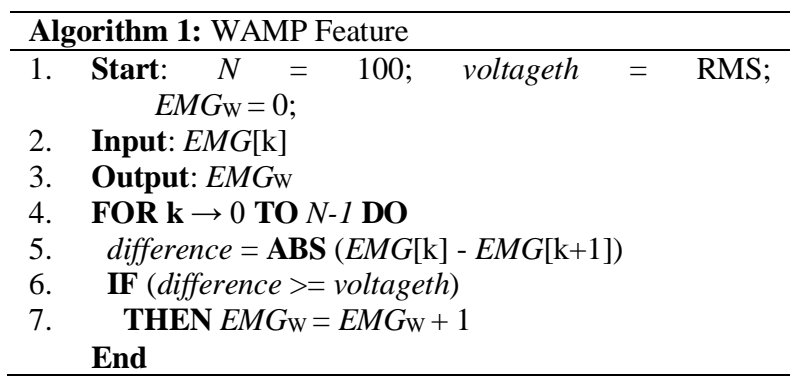

$$
\begin{aligned}
& E M G_{W}=\sum_{i=1}^{100}\left[f\left(\left|E M G_{i}-E M G_{i+1}\right|\right)\right] \\
& f(x)=\left\{\begin{array}{l}
1, \text { if } \rightarrow x \geq \text { threshold } \\
0, \text { otherwise }
\end{array}\right.
\end{aligned}
$$


where $E M G_{W}$ is the WAMP parameter obtained each of 100 samples, EMGi is the raw EMG signal for the i-samples, and The threshold shall be the amplitude needed to pass the signal to be registered. The WAMP Parameter Implementation was determined in accordance with Algorithm 1. The $E M G_{W}$ can represent an elbow-joint angle approximation. Generally, the threshold value was pre-defined with the values between 0.05 and $0.3 \mathrm{mV}$ as mentioned by previous studies [17]. The EMG signal-based threshold was produced in this study. Next, the threshold values were obtained from RMS. The next stage was to standardize the parameter of EMGW using (2).

$$
E M G_{N}=\frac{E M G_{W}-E M G_{W(M I N)}}{E M G_{W(M A X)}-E M G_{W(M I N)}}
$$

where $E M G_{N}$ is the normalized WAMP features, $E M G_{W}$ is the WAMP parameter that is obtained from feature extraction process, $E M G_{W(M I N)}$ is the minimum value of the parameter, and $E M G_{W(M A X)}$ is the maximum value of the parameter. Because of the raw EMG data, an absolute function was used before the extraction of the element, so that the $E M G_{W}$ minimum value was always zero. In the offline data processing, the maximum of $E M G_{\mathrm{W}}$ was obtained by selecting the data for every cycle. Further, in the real-time data processing, the maximum of the $E M G_{\mathrm{W}}$ may be obtained by conducting some of trial before performing the proposed model. In this stage, the normalized WAMP parameter can represent the elbow-joint angle estimation with some ripples and noises. Therefore, to smooth the $E M G_{N}$, it needs a filtering process as recommended by previous researchers [10]. The LPF was produced digitally, based on Infinite Impulse Responses (IIR). The LPF equation was shown in (3).

$$
\begin{aligned}
E M G_{L}[k]= & b_{0} E M G_{N}[k]+b_{1} E M G_{N}[k-1]+b_{2} E M G_{N}[k-2] \\
& -a_{1} E M G_{L}[k-1]-a_{2} E M G_{L}[k-2]
\end{aligned}
$$

where $E M G_{L}[k]$ is the filtered features for $k$-th sample. The $a_{1}, a_{2}, b_{0}, b_{1}$ and $b_{2}$ are the LPF coefficients. The LPF cut-off frequency was chosen from $80 \mathrm{~Hz}$ to $100 \mathrm{~Hz}$ to get the best elbow-joint angle estimate.

The $E M G_{L}[\mathrm{k}]$ was the estimated angle with the normalized condition (ranged between 0 and 1). The actual angle was calculated in this analysis using a potentiometer that was used to test and calibrate the projected angle. The estimated angle is obtained by using two parameters to calibrate the output of the filtered functions, the maximum value of the measured angle $\left(A_{M E A(M A X)}\right)$ and the gain constant $(G)$ as written in (4).

$$
A_{E S T}[k]=G \cdot A_{M E A(M A X)} \cdot E M G_{L}[k]
$$

where $A_{E S T}[\mathrm{k}]$ is the estimated angle, $G$ is an adjustable constant to get the best output of the projected angle, $A_{M E A}(M A X)$ is the maximum value of the measured angle which is obtained from the maximum value of the movement of the elbow joint and $E M G_{L}[\mathrm{k}]$ is obtained from (3). Figure 2 outlines steps of the process of tuning and calibration. The tuning technique was used to achieve maximum efficiency of calculation of the elbow-joint angle. The calibration stage is for the determined angle to be tested at the measured angle. The root mean square (RMS) processor used to produce a dynamic threshold for the WAMP features is the main part of this block diagram. The RMS processor calculated the energy of the EMG signal (5) so that threshold values can adapt to any variation of the EMG amplitude for different loads so that the accuracy of the estimation can be maintained.



Figure 2. The tuning and calibration process to achieve optimum elbow-joint angle estimation efficiency 


$$
R M S=\sqrt{\frac{1}{N} \sum_{i=1}^{N} E M G_{i}}
$$

where $E M G_{i}$ is the $i$-th sample and $N$ is windows length for the feature extraction process.

$$
\text { Threshold }=\text { C.RMS }
$$

The WAMP threshold was determined based on (6). The $C$ constant was chosen in such a way that the performance is the highest. The $C$ constant was chosen between $60 \%$ and $80 \%$ of the RMS value by experiments. The calibration depended on the measured maximum-angle $\left(A_{\mathrm{MEA}(\mathrm{MAX})}\right)$ and gain factor $(G)$. In this work, the $A_{\mathrm{MEA}(\mathrm{MAX})}$ was chosen according to the experimental protocol in which the range of motion is between $0^{\circ}$ and $145^{\circ}$ Figure 1 , therefore the $A_{\text {MEA(MAX) }}$ equal to 145 . Gain factor $(G)$ was selected between 1.0 and 2.0. These values were determined by experiments in the first measurement of the EMG signal.

\section{RESULTS}

This section presented the result of this study. The EMG activity, result of the feature extraction using WAMP parameter with an adaptive threshold. Statistical analysis was also shown in order to get the performance comparison between parameters.

\subsection{Estimate the position}

Figure 3 shows an example of the estimation from the subject. Figure 3(a) shows the surface EMG signal activities were based on the elbow joint angle movement. Following the extraction process, the characteristics yielded may match the position of the elbow Figure 3(c). Figure 4(d) shows that the predicted elbow joint angles. In this example, the RMSE of the estimated angle was $10.67^{\circ}$ and Pearson's correlation coefficient was 0.983. Another example, in Figure 4, was the approximate elbow joint angle for a cyclic random motion from subject A. In this case, RMS error and correlation were $13.38^{\circ}$ and 0.958 , respectively.
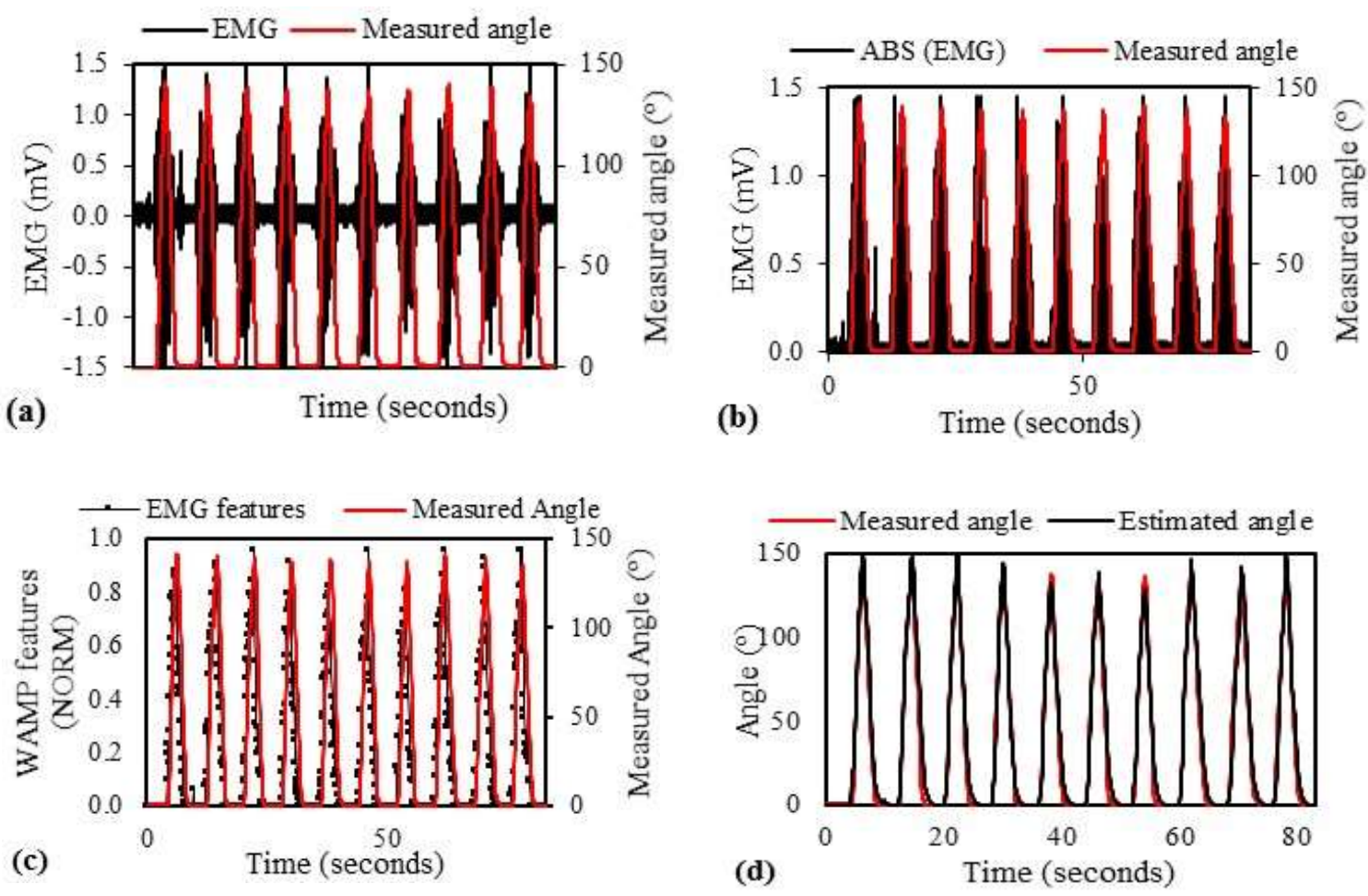

Figure 3. (a) original of raw electromyography signal, (b) rectifying of EMG, (c) EMG feature, (d) smoothing feature 



Figure 4. (a) original of raw electromyography (b) rectifying of EMG signal, (c) The EMG features, (d) The smoothing feature

\subsection{Effect of independent variable on performance}

Figure 5 and Figure 6 showed the estimated performance results (RMS error and correlation) for all subjects. Each type of motion and period has a different performance (RMS error and correlation coefficient). As shown in Table 1, the mean RMS error for a single trial was 9.78 \pm 0.86 . Table 1 presents that the mean RMS error for the continuous trial was $10.33^{\circ} \pm 0.46^{\circ}$ Table 1 . The mean RMS error for the random trial was $15.35^{\circ}$ $\pm 2.21^{\circ}$. For a single trial the mean Pearson correlation coefficient was $0.98 \pm 0.004$ Table 2 . For the continuous trial the mean correlation coefficient was $0.97 \pm 0.003$ Table 2 . For the random trial the mean correlation coefficient (mean \pm standard deviation) was $0.94 \pm 0.017$ Table 2 . The test showed that the RMSE was normally distributed $(\mathrm{p}>0.05)$.

From the ANOVA single-factor statistical analysis Table 1 and Table 2, It was shown that there was no significant difference between the coefficient of correlation ( $p>0.05$ ) between the movement periods. (P1: 6 seconds, P2: 8 seconds, and P3: 10 seconds) in the single and continuous tests.
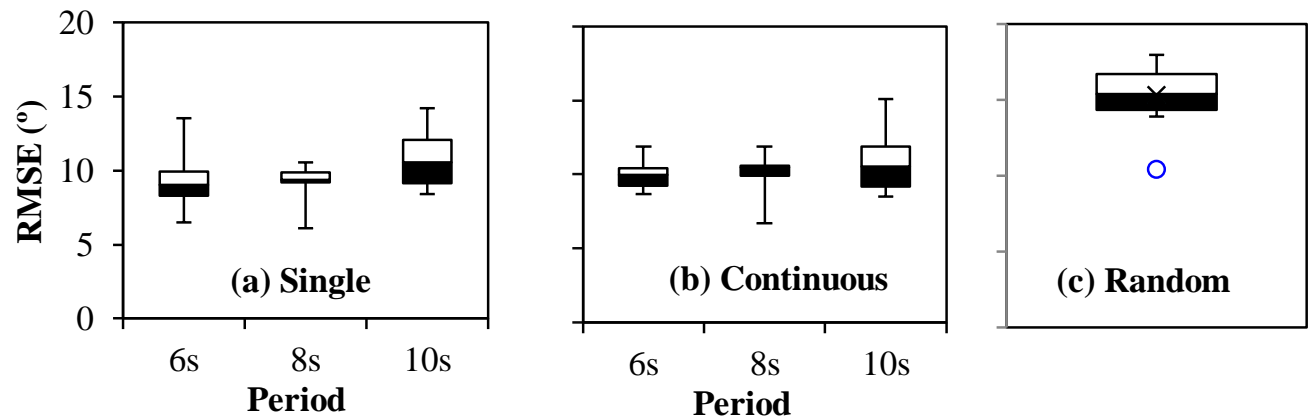

Figure 5. The boxplot of the variance of RMSE that classified based on the cycle of motion and type of motion: (a) single, (b) continuous, (c) random) for all subjects (10 participants) 

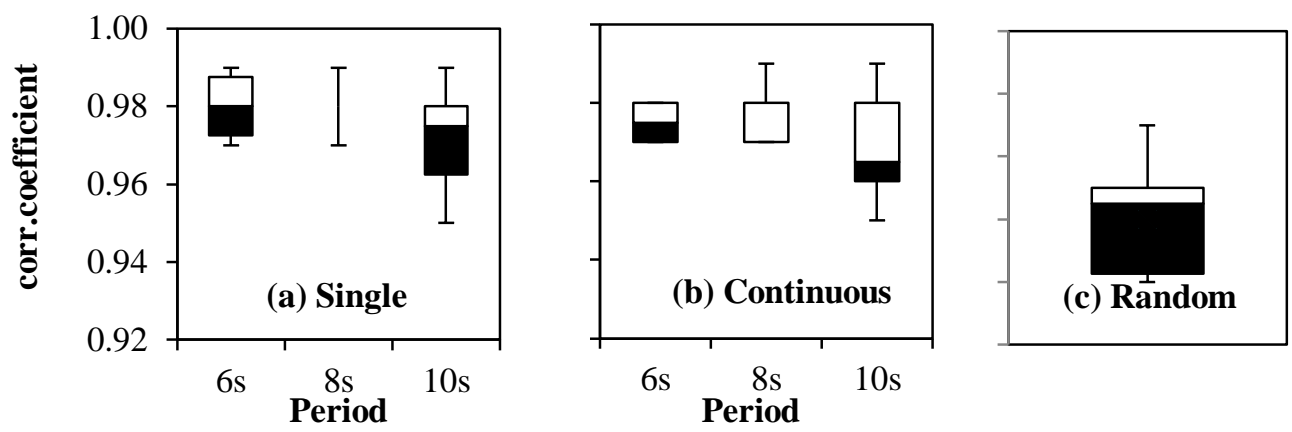

Figure 6. The boxplot of the variance of mean correlation coefficient that classified based on the cycle of motion and type of motion: (a) single, (b) continuous, (c) random) for all subjects (10 participants)

Table 1. Overview of coefficient of correlation for single, continuous, random process trial

\begin{tabular}{|c|c|c|c|c|c|c|c|}
\hline \multirow[t]{2}{*}{ Subject } & \multicolumn{3}{|c|}{ Single Cycle } & \multicolumn{3}{|c|}{ Continuous Cycle } & \multirow{2}{*}{$\begin{array}{l}\text { Random } \\
\text { Cycle }\end{array}$} \\
\hline & Period: $6 \mathrm{~s}$ & Period: $8 \mathrm{~s}$ & Period: $10 \mathrm{~s}$ & Period: $6 \mathrm{~s}$ & Period: $8 \mathrm{~s}$ & Period: $10 \mathrm{~s}$ & \\
\hline $\mathrm{A}$ & $0.99 \pm 0.011$ & $0.98 \pm 0.010$ & $0.98 \pm 0.014$ & $0.98 \pm 0.004$ & $0.98 \pm 0.007$ & $0.98 \pm 0.015$ & $0.97 \pm 0.009$ \\
\hline B & $0.98 \pm 0.004$ & $0.98 \pm 0.005$ & $0.98 \pm 0.003$ & $0.98 \pm 0.006$ & $0.98 \pm 0.005$ & $0.98 \pm 0.005$ & $0.95 \pm 0.006$ \\
\hline $\mathrm{C}$ & $0.97 \pm 0.006$ & $0.98 \pm 0.008$ & $0.95 \pm 0.016$ & $0.97 \pm 0.004$ & $0.97 \pm 0.008$ & $0.96 \pm 0.013$ & $0.92 \pm 0.015$ \\
\hline $\mathrm{D}$ & $0.98 \pm 0.004$ & $0.98 \pm 0.006$ & $0.97 \pm 0.01$ & $0.97 \pm 0.012$ & $0.97 \pm 0.007$ & $0.96 \pm 0.007$ & $0.93 \pm 0.009$ \\
\hline $\mathrm{E}$ & $0.97 \pm 0.014$ & $0.97 \pm 0.013$ & $0.96 \pm 0.018$ & $0.97 \pm 0.008$ & $0.97 \pm 0.01$ & $0.96 \pm 0.008$ & $0.94 \pm 0.022$ \\
\hline $\mathrm{F}$ & $0.99 \pm 0.005$ & $0.98 \pm 0.004$ & $0.98 \pm 0.016$ & $0.98 \pm 0.004$ & $0.98 \pm 0.004$ & $0.97 \pm 0.008$ & $0.95 \pm 0.009$ \\
\hline $\mathrm{G}$ & $0.97 \pm 0.009$ & $0.98 \pm 0.008$ & $0.97 \pm 0.014$ & $0.97+0.013$ & $0.97 \pm 0.013$ & $0.96 \pm 0.008$ & $0.95 \pm 0.012$ \\
\hline $\mathrm{H}$ & $0.99 \pm 0.003$ & $0.99 \pm 0.002$ & $0.99 \pm 0.007$ & $0.98 \pm 0.01$ & $0.99 \pm 0.003$ & $0.99 \pm 0.003$ & $0.95 \pm 0.007$ \\
\hline I & $0.98+0.005$ & $0.97 \pm 0.009$ & $0.98 \pm 0.005$ & $0.98+0.005$ & $0.97 \pm 0.009$ & $0.98 \pm 0.005$ & $0.92 \pm 0.015$ \\
\hline $\mathrm{J}$ & $0.98 \pm 0.006$ & $0.98 \pm 0.008$ & $0.96 \pm 0.01$ & $0.97 \pm 0.008$ & $0.97 \pm 0.004$ & $0.95 \pm 0.01$ & $0.92 \pm 0.01$ \\
\hline \multirow{2}{*}{$\begin{array}{l}\text { Average } \\
\text { Total Av. }\end{array}$} & $0.98 \pm 0.008$ & $0.98 \pm 0.006$ & $0.97 \pm 0.012$ & $0.98 \pm 0.005$ & $0.98 \pm 0.007$ & $0.97 \pm 0.013$ & \multirow{2}{*}{$0.94 \pm 0.017$} \\
\hline & & $0.98 \pm 0.004$ & & & $0.97 \pm 0.003$ & & \\
\hline $\mathrm{p}$-value & & 0.1216 & & & 0.6965 & & - \\
\hline
\end{tabular}

Table 2. RMSE description for single, continuous, random cycle trial (RMSE in grades)

\begin{tabular}{|c|c|c|c|c|c|c|c|}
\hline \multirow[t]{2}{*}{ Subject } & \multicolumn{3}{|c|}{ Single Cycle } & \multicolumn{3}{|c|}{ Continuous Cycle } & \multirow{2}{*}{$\begin{array}{c}\text { Random } \\
\text { Cycle }\end{array}$} \\
\hline & Period: $6 \mathrm{~s}$ & Period: $8 \mathrm{~s}$ & Period: $10 \mathrm{~s}$ & Period: $6 \mathrm{~s}$ & Period: $8 \mathrm{~s}$ & Period: $10 \mathrm{~s}$ & \\
\hline $\mathrm{A}$ & $8.13 \pm 3.2$ & $9.29 \pm 2.81$ & $8.43 \pm 3.26$ & $9.95 \pm 1.09$ & $10.53 \pm 2.17$ & $10.04 \pm 3.11$ & $10.48 \pm 1.71$ \\
\hline B & $8.73 \pm 1.32$ & $10.05 \pm 1.85$ & $9.28 \pm 1.25$ & $9.67 \pm 2.31$ & $9.68 \pm 2.16$ & $8.5 \pm 1.55$ & $13.90 \pm 2.12$ \\
\hline $\mathrm{C}$ & $10.07 \pm 1.2$ & $8.06 \pm 1.83$ & $13.37 \pm 2.29$ & $11.27 \pm 0.73$ & $10.7 \pm 2.11$ & $12.89 \pm 1.8$ & $16.21 \pm 1.31$ \\
\hline $\mathrm{D}$ & $9.00 \pm 1.28$ & $9.20 \pm 0.71$ & $11.07 \pm 2.43$ & $10.26 \pm 1.34$ & $10.05 \pm 1.25$ & $11.74 \pm 1.32$ & $18.01 \pm 1.05$ \\
\hline $\mathrm{E}$ & $9.46 \pm 2.32$ & $9.44 \pm 1.83$ & $11.35 \pm 2.78$ & $10.45 \pm 0.93$ & $10.37 \pm 1.28$ & $11.0 \pm 1.34$ & $15.38 \pm 2.37$ \\
\hline $\mathrm{F}$ & $8.12 \pm 1.41$ & $9.30 \pm 1.18$ & $10.03 \pm 3.49$ & $8.67 \pm 0.84$ & $9.86 \pm 0.64$ & $11.91 \pm 1.37$ & $15.43 \pm 1.41$ \\
\hline $\mathrm{G}$ & $13.55 \pm 2.01$ & $10.53 \pm 2.44$ & $12.31 \pm 3.51$ & $9.95 \pm 1.09$ & $10.53 \pm 2.17$ & $10.04 \pm 3.11$ & $15.13 \pm 1.70$ \\
\hline $\mathrm{H}$ & $6.47 \pm 0.98$ & $6.13 \pm 1.12$ & $9.09 \pm 2.61$ & $9.11 \pm 2.17$ & $6.7 \pm 0.77$ & $8.92 \pm 1.05$ & $14.11 \pm 1.06$ \\
\hline I & $9.09 \pm 1.10$ & $10.58 \pm 1.91$ & $8.48 \pm 1.36$ & $9.09 \pm 1.1$ & $10.58 \pm 1.91$ & $8.48 \pm 1.36$ & $17.93 \pm 1.16$ \\
\hline $\mathrm{J}$ & $10.96 \pm 0.66$ & $9.44 \pm 1.81$ & $14.24 \pm 1.67$ & $11.91 \pm 1.37$ & $11.86 \pm 0.66$ & $15.09 \pm 1.25$ & $16.88 \pm 1.14$ \\
\hline Average & $9.36 \pm 1.9$ & $9.2 \pm 1.3$ & $10.77 \pm 2.05$ & $10.03 \pm 1.00$ & $10.09 \pm 1.33$ & $10.86 \pm 2.11$ & \\
\hline Total Av. & & $9.78 \pm 0.86$ & & & $10.33 \pm 0.46$ & & $15.35 \pm 2.21$ \\
\hline p-value & & 0.1163 & & & 0.4647 & & - \\
\hline
\end{tabular}

\section{DISCUSSION}

The elbow joint angle could be calculated in this analysis with a non-pattern recognition which is a non-machine learning process by extracting the EMG signal from biceps. The proposed method could adapt to varying period of motion with no significant difference of the performance $(p>0.05)$.

\subsection{EMG activity}

In this study, the EMG signal produced from biceps was more powerful than the signal from triceps. The marginal action of the triceps has been identified by past researchers. Previous researchers revealed in the sagittal plane of the elbow joint movement that the EMG signal produced from triceps showed negligible activity than that from biceps [18]. For this reason, the EMG signal produced from biceps was the only one concerned in this study to estimate the elbow joint angle. Figure 5 and Figure 6 display the RMSE variance for all topics in the box-plot diagram. The RMSE of the measured angle revealed a broader variation in the motion 
time of 10 seconds (the standard deviation was $2.00^{\circ}$ to $2.74^{\circ}$ ) if it is compared to that of 6 seconds and 8 seconds (the standard deviation was $0.98^{\circ}$ to $1.8^{\circ}$ ). This could be caused by a little activity of EMG signal when the movement was slow [19]. The best mean RMSE was obtained in the single-cycle trial for all of the period, loads and subjects within ranged between $9.10^{\circ} \pm 0.66^{\circ}$ and $10.64^{\circ} \pm 1.73^{\circ}$. By increasing the complexities of the movement, in the random trial, the accuracy of the estimation was lower than that of single-cycle trial and continuous cycle trial. Pau [5] also found that the performance was decreased when the motion was more complex. The correlation coefficients for all variables (movement periods and types of movements) show, however, that the coefficients were higher than 0.9 (Table 1). This indicates that the method proposed is highly correlated with the angle measured.

\subsection{Estimation}

The proposed model revealed that the estimate has a consistency $(\mathrm{p}>0.05)$ for all variables (subjects, movement period and movement type) using only the EMG signal. This was due to the fact that the WAMP threshold was obtained for each window duration based on the EMG intensity so that the EMG signal function could match the estimate. The proposed model is comparable with several previous studies in the same setting, which uses only the EMG signal for the estimation. Pau et al. proposed an estimation using myoelectric signal (from biceps and triceps) based on Hill musculoskeletal model[5]. The mean RMSE values obtained are $6.53^{\circ} \pm 3.2^{\circ}$ for a single cycle of motion, $22.0^{\circ} \pm 6.6^{\circ}$ for continuous of motion and $22.4^{\circ} \pm 5.0^{\circ}$ for random motion. However, in their study, to obtain the maximum performance, they optimized the performance using a Genetic algorithm which was a time-consuming process to obtain local minima. Tang et al. developed EMG to angle model that recognized a single cycle of motion (flexion and extension) with varying loads using the artificial neural network (ANN) [6]. The EMG signal was derived from four muscles and used as input to the ANN. The RMSE values were $7.86^{\circ} \pm 1.14^{\circ}$ for intra-load (testing with the same load) and $26.73^{\circ} \pm 9.01^{\circ}$ for inter-load (testing with the other load). The performance improved after they integrated the EMG signal and a force sensor as inputs to the ANN. Ko and Mak reported a difference accuracy (RMSE) between loaded and unloaded trials for flexion motion which is $13.71^{\circ} \pm 5.89^{\circ}$ and $34.64^{\circ} \pm 7.79^{\circ}$ respectively[20]. Triwiyanto et al. suggested the Kalman filter for estimation [21]. The RMSE values were ranged between $9.41^{\circ} \pm 2.53^{\circ}$ and $15.02^{\circ} \pm 2.14^{\circ}$ for three periods of motion $(6 \mathrm{~s}, 8 \mathrm{~s}$, and $12 \mathrm{~s})$.

\subsection{Effect of independent variable on performance}

The performance of this proposed method depended on how the individual performed the motion in the direction of flexion and extension according to the experimental protocol. In this situation, the participants must not perform any other contraction (such as pronation and supination) besides the flexion and extension motion. Even though we have homogenized the participants but still each participant has its own EMG characteristic (the amplitude and pattern). This proposed approach was limited only to the identification of one degree of elbow-joint freedom (1 DOF). A new method needs developing so that it can recognize more than one DOF. Therefore, it needs an investigation of the muscle group that was related to the movements and motion direction of the limb. Muscle fatigue is a condition to be further addressed in the data collection process, as Basmajian and de Luca reported that muscle fatigue affected spectral parameters of surface electromyography signal $[22,23]$. In this analysis, the muscle was assumed to be in a non-fatigue state. Therefore, to prevent muscle tiredness, the subjects were given some rest time in the data collection process for each trial. Though there have been many attempts to account for the impact of muscle tiredness [24, 25]. The creation of a model that can compensate for muscle tiredness in dynamic motion remains a challenging problem.

\section{CONCLUSION}

The main objective of this study is to build an electromyography signal method for predicting the elbow position based on non-machine learning, which directly uses the extraction and filtering technique in the time-domain function. We suggested that the threshold value be generated on the basis of the EMG energy (RMS) so that the model can be adapted to any changes in subjects and variables such movement periods and movement types. The effectiveness of the proposed method has statistically examined and it showed a good performance for a single, continuous and random movement with varying periods of motion. The statistical analysis showed that, for all subjects with different motion periods, there was mostly no significant difference in RMSE. The tuning method was achieved in a simple fashion by changing the constant $\mathrm{G}$ and $\mathrm{C}$. In the future study, the approach can be expanded to include more degrees of freedom and the prosthetic or exoskeletal tools can be used as a control signal. 


\section{REFERENCES}

[1] T. Triwiyanto, et al., "Embedded system for upper-limb exoskeleton based on electromyography control," TELKOMNIKA, vol. 17, no. 6, pp. 2992-3002, 2019.

[2] M. Asghari Oskoei and H. Hu, "Myoelectric control systems-A survey," Biomed. Signal Process. Control, vol. 2, no. 4, pp. 275-294, 2007.

[3] Q. Li, Y. Song, Z. Hou, and B. Zhu, "sEMG based joint angle estimation of lower limbs using LS-SVM," Lect. Notes Comput. Sci. (including Subser. Lect. Notes Artif. Intell. Lect. Notes Bioinformatics), vol. 8226 LNCS, no. PART 1, pp. 292-300, 2013.

[4] S. Guo, M. Pang, B. Gao, H. Hirata, and H. Ishihara, "Comparison of sEMG-Based feature extraction and motion classification methods for upper-limb movement," Sensors (Basel)., vol. 15, no. 4, pp. 9022-9038, 2015.

[5] J. W. L. Pau, S. S. Q. Xie, and A. J. Pullan, "Neuromuscular interfacing: Establishing an EMG-driven model for the human elbow joint," IEEE Trans. Biomed. Eng., vol. 59, no. 9, pp. 2586-2593, 2012.

[6] Z. Tang, H. Yu, and S. Cang, "Impact of load variation on joint angle estimation from surface emg signals," IEEE Trans. Neural Syst. Rehabil. Eng., vol. PP, no. 99, p. 1, 2015.

[7] J. Too, et al., "Application of gabor transform in the classification of myoelectric signal," TELKOMNIKA, vol. 17, no. 2, pp. 873-881, 2019.

[8] T. D. Lalitharatne, et al., "Evaluation of fuzzy-neuro modifiers for compensation of the effects of muscle fatigue on emg-based control to be used in upper-limb power-assist exoskeletons," J. Adv. Mech. Des. Syst. Manuf., vol. 7, no. 4, pp. 736-751, 2013.

[9] S. Lee, H. Kim, H. Jeong, and J. Kim, "Analysis of musculoskeletal system of human during lifting task with arm using electromyography," Int. J. Precis. Eng. Manuf., vol. 16, no. 2, pp. 393-398, 2015.

[10] G. Jang, J. Kim, Y. Choi, and J. Yim, "Human shoulder motion extraction using EMG signals," Int. J. Precis. Eng. Manuf., vol. 15, no. 10, pp. 2185-2192, 2014.

[11] H. J. Yu, A. Y. Lee, and Y. Choi, "Human elbow joint angle estimation using electromyogram signal processing," IET Signal Process., vol. 5, no. 8, p. 767, 2011.

[12] C. J. De Luca, “Surface Electromyography : Detection and Recording,” DelSys Inc., vol. 10, no. 2, pp. 1-10, 2002.

[13] Martini, Fundamental of Anatomy and Physiology, 9th ed. Boston: Pearson Education, 2012.

[14] T. Triwiyanto, et al., "Evaluating the performance of Kalman filter on elbow joint angle prediction based on electromyography," Int. J. Precis. Eng. Manuf., vol. 18, no. 12, pp. 1739-1748, Dec. 2017.

[15] E. F. Shair, S. A. Ahmad, A. R. Abdullah, M. H. Marhaban, and S. B. Mohd Tamrin, "Determining best window size for an improved gabor transform in EMG signal analysis," TELKOMNIKA, vol. 16, no. 4, pp. 1650-1658, 2018.

[16] Triwiyanto, O. Wahyunggoro, H. A. Nugroho, and Herianto, "An investigation into time domain features of surface electromyography to estimate the elbow joint angle," Adv. Electr. Electron. Eng., vol. 15, no. 3, pp. 448-458, 2017.

[17] D. Wu, X. Sun, Z. Zhang, Z. Du, and L. Sun, "Quantitative relationship modeling between surface electromyography and elbow joint angle," no. Bmei, pp. 1002-1006, 2010.

[18] T. Lenzi, S. M. M. De Rossi, N. Vitiello, and M. C. Carrozza, "Intention-Based EMG control for powered exoskeletons," IEEE Trans. Biomed. Eng., vol. 59, no. 8, pp. 2180-2190, Aug. 2012.

[19] B. E. Mustard and R. G. Lee, "Relationship between EMG patterns and kinematic properties for flexion movements at the human wrist," Exp. Brain Res., vol. 66, no. 2, pp. 247-256, Apr. 1987.

[20] T. K. K. Koo and A. F. T. Mak, "Feasibility of using EMG driven neuromusculoskeletal model for prediction of dynamic movement of the elbow," J. Electromyogr. Kinesiol., vol. 15, no. 1, pp. 12-26, 2005.

[21] T. Triwiyanto, et al., "Evaluating the performance of Kalman filter on elbow joint angle prediction based on electromyography,” Int. J. Precis. Eng. Manuf., vol. 18, no. 12, pp. 1739-1748, 2017.

[22] J. V Basmajian and C. J. de Luca, "Chapter 8. muscle fatigue and time-dependent parameters of the surface EMG Signal," Muscles alive: their functions revealed by electromyography, Baltimore: Williams \& Wilkins, pp. 201-222, 1985.

[23] Triwiyanto, et al., "Continuous wavelet transform analysis of surface electromyography for muscle fatigue assessment on the elbow joint motion," Adv. Electr. Electron. Eng., vol. 15, no. 3, 2017.

[24] E. Park and S. G. Meek, "Fatigue compensation of the electromyographic signal for prosthetic control and force estimation," IEEE Trans. Biomed. Eng., vol. 40, no. 10, pp. 1019-1023, 1993.

[25] Y. Na and J. Kim, "Dynamic elbow flexion force estimation through a muscle twitch model and sEMG in a fatigue condition,” IEEE Trans. Neural Syst. Rehabil. Eng., vol. 4320, no. 99, pp. 1-1, 2016.

\section{BIOGRAPHIES OF AUTHORS}

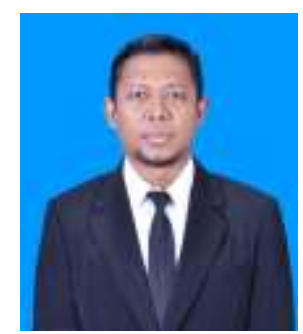

Triwiyanto received B.S. degree in Physics at Airlangga University in 1997, and received M.S. degree in Electronic Engineering in Institute of Technology Sepuluh Nopember, Surabaya, Indonesia, in 2004. He received a Ph.D degree in Electrical Engineering at Gadjah Mada University, Yogyakarta, Indonesia, in 2018; and since 1998 he is a lecturer at the Department of Electromedical Engineering, POLTEKKES KEMENKES Surabaya, Indonesia. His recent research interests include biomedical signal analysis, machine learning, embedded system, electronic instrumentation, assistive and rehabilitation devices. 

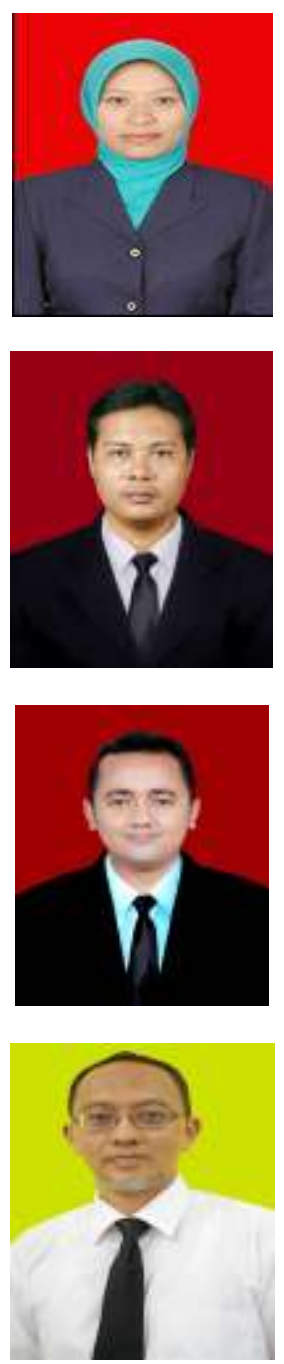

Triana Rahmawati received B.S. degree in Physics Engineering at Institute of Technology Sepuluh Nopember in 2008, and received M.S. degree in Electronic Engineering in Gadjah Mada University, Yogyakarta, Indonesia, in 2013; and since 2008 she is a lecturer at The Department of Electromedical Engineering, POLTEKKES KEMENKES SURABAYA, Indonesia. Her recent research interests include Electromedical Instrumentation, biomedical signal analysis, Artificial Intelligence and machine learning.

Endro Yulianto received B.S. degree in Nuclear Engineering at Gadjah Mada University in 1999, and received M.S. degree in Electronic Engineering in Institute of Technology Sepuluh Nopember, Surabaya, Indonesia, in 2004. He received a Ph.D degree in Electrical Engineering at Gadjah Mada University, Yogyakarta, Indonesia, in 2013; and since 1999 he is a lecturer at the Department of Electromedical Engineering, POLTEKKES KEMENKES Surabaya, Indonesia. His recent research interests include biomedical signal processing, machine learning, electronic instrumentation, assistive and rehabilitation devices.

Muhammad Ridha Mak'ruf received B.S. degree in Physics Engineering at Institute of Technology Sepuluh Nopember in 2005, and received M.S. degree in Biomedical Engineering in Universitas Indonesia, in 2012. Since 2013 he is a lecturer at the Department of Electromedical Engineering, POLTEKKES KEMENKES Surabaya, Indonesia. His recent research interests include biomedical signal analysis, machine learning, embedded system, electronic instrumentation, and image processing.

Priyambada Cahya Nugraha received B.S. degree in Electrical Engineering (Electronic Engineering) at Institut Teknologi Sepuluh Nopember (ITS), Surabaya, Indonesia, in 1994, and received M.S. degree in Electrical Engineering (Biomedical Engineering) in Institut Teknologi Bandung, Bandung, Indonesia, in 2003, and since 1998 he is a lecturer at the Department of Electromedical Engineering, POLTEKKES KEMENKES Surabaya, Indonesia. His recent research interests include biomedical sensor, electronic instrumentation, biomedical signal processing $\&$ analysis, microcontroller and telemedicine devices. 